\title{
Synthesis, characterization and catalytic activity of ordered SBA-15 materials containing high loading of diamine functional groups
}

\author{
Xueguang Wang, Jerry C.C. Chan, Yao-Hung Tseng, Soofin Cheng * \\ Department of Chemistry, National Taiwan University, Taipei 106, Taiwan
}

Received 4 November 2005; received in revised form 3 May 2006; accepted 5 May 2006

Available online 21 June 2006

\begin{abstract}
Diamine-functionalized mesoporous SBA-15 materials have been synthesized by co-condensation of tetraethylorthosilicate (TEOS) with [3-(2-aminoethyl aminopropyl)]trimethoxysilane (ATMS) using amphiphilic block copolymer P123 as pore-directing agent under the aid of inorganic salt $\mathrm{NaCl}$ and with prehydrolysis of TEOS prior to the addition of organosiloxane. The functionalized materials have hexagonal mesostructure ordering, narrow pore-size distribution with diameter around $60 \AA$, and high loadings of amino groups (up to $3.49 \mathrm{mmol} \mathrm{g}^{-1}$ ). Prehydrolysis of TEOS was a key step for preparation of well-ordered mesoporous materials with amino functionalities. Inorganic salt had great influence on the ordering of mesostructure and the morphology of the resultant materials. The diamine-functionalized materials showed better catalytic performance than the aminopropyl-functionalized counterpart in the Knoevenagel reaction of benzaldehyde with ethyl cynoacetate to form $\alpha, \beta$-unsaturated compound in liquid phase.
\end{abstract}

(c) 2006 Elsevier Inc. All rights reserved.

Keywords: SBA-15; Aminoethylaminopropyl; Mesostructure; Co-condensation; Functionalization; Knoevenagel reaction

\section{Introduction}

Since the discovery of the M41S class of mesoporous silicates [1], incorporation of organic groups to impart functionalities to the pore surface of the mesoporous silica materials has attracted much attention due to the potential applications of the resultant materials in the field of catalysis [2-5], separation [6,7], sensor design [8], and nanoscience $[9,10]$. There are two approaches for surface functionalization, i.e. grafting (also known as post-synthesis) and direct synthesis or co-condensation [11]. In postsynthesis, organic functional groups are grafted through the reaction of a silane coupling agent with the free and germinal silanol groups on the surface of mesopores. The organic functional groups can be easily chosen and designed to meet different requirements. The resultant materials generally maintain highly ordered structures

\footnotetext{
${ }^{*}$ Corresponding author. Tel.: +8862 23638017; fax: +886223636359. E-mail address: chem1031@ntu.edu.tw (S. Cheng).
}

and show relatively high hydrothermal stability after grafting reaction [12]. However, the distribution of the functional groups on the surface of the pore wall is likely not uniform and the organic groups are grafted mainly on the external surface of the mesoporous particles or near the pore mouth because of the mass transfer [13]. Comparatively, the direct synthesis pathway by co-condensation of siloxane and organosiloxane precursors often offers a better control of the resultant materials in terms of a higher and more uniform surface coverage of the organic functionalities without the blockage of the mesopores [14]. Nevertheless, the resultant materials usually show less structural ordering than the pure siliceous counterpart, and the organosiloxane precursors must be carefully chosen to avoid possible phase separation and $\mathrm{Si}-\mathrm{C}$ bond cleavage during the synthesis and surfactant removal processes [10]. Up to date, just several of the organic functional groups can be synthesized through the direct co-condensation method.

There has been considerable interest in the development of heterogeneous solid catalysts, since the use of heterogeneous 
catalytic processes allows easier separation, recovery and recycling of the catalyst from the reaction mixture. Moreover, heterogeneous catalysts sometimes gave better selectivities than the homogeneous ones in many bimolecular reactions [15]. Amino-functionalized mesoporous silicas have been widely investigated due to the applications in base-catalyzed reactions [14,16]. However, most of the work was on the modification of MCM-type [17,18], and HMS-type [19] materials with relatively small mesopore sizes, which were synthesized under basic or neutral conditions. In the large mesoporous silica system, disordered materials were often obtained by direct co-condensation of tetraethylorthosilicate (TEOS) and amino-functional silane due to the interference of the protonated amine groups in the self-assembly of the copolymer surfactant and the silica precursor under the strong acidic condition $[20,21]$. However, functionalized mesoporous materials with large pores are desired in many applications such as the immobilization and encapsulation of large molecules $[21,22]$. Recently, we have successfully synthesized largepore ordered SBA-15 silica functionalized with a high loading of aminopropyl groups via prehydrolysis of TEOS prior to the addition of APTES $[23,24]$. Moreover, in the attempt to incorporate more hydrophobic functional groups such as $N$-methylaminopropyl groups, the addition of inorganic salts in the synthesis mixture was found to significantly improve the crystallographic ordering of the mesoporous silica [25]. The salt effect in the synthesis of mesoporous materials has been reported to improve the hydrothermal stability [26], control the morphology [27], tailor the framework porosity [28], and improve the mesostructure ordering [29].

Diamine-functionalized mesoporous materials are attracting much attention due to the strong absorbing and chelating performance of ethylenediamine (ED) groups. Dai et al. [6] synthesized diamine-functionalized ordered mesoporous materials to selectively absorb the targeted metal ions based on the affinity of the surface-coated functional ligand for a specific metal ion. Zhang et al. [30] successfully prepared transition metal oxide or sulfide nanoparticles in ordered mesoporous silicas with ethylenediamine groups by post-synthesis. More recently, controlled $\mathrm{Pt}$ and $\mathrm{Au}$ nanoparticles in ordered mesoporous materials have also been reported using diamine groups on the pore surface as stabilizer [31-33]. Even more interestingly, mesoporous ethane-silicas functionalized with diaminocyclohexane showed a high catalytic activity and the enantioselectivity in the asymmetric transfer hydrogenation of acetophenone [34]. These urges us to report large-pore ordered silica containing diamine groups using triblock copolymer Pluronic P123 $\left(\mathrm{EO}_{20} \mathrm{PO}_{70} \mathrm{EO}_{20}\right)$ as pore-directing agent under a strong acidic condition through the combination of TEOS prehydrolysis and inorganic salt, which is expected to have potential applications in the preparation of controlled high loading metal or metal oxide nanoparticles in the mesoporous silica and to have better catalytic activity in solid base catalytic reactions.

\section{Experimental}

Surfactant P123 $\left(\mathrm{EO}_{20} \mathrm{PO}_{70} \mathrm{EO}_{20}, M_{\mathrm{av}} \sim 5800\right)$ and [3(2-aminoethyl)aminopropyl] trimethoxysilane (ATMS, 97\%) were purchased from Aldrich. Other chemicals were from Acros. All chemicals were used as received.

\subsection{Synthesis of materials}

Diamine-functionalized SBA-15 materials were prepared through co-condensation method. In the typical synthesis, $4 \mathrm{~g}$ of Pluronic 123 and $11 \mathrm{~g}$ of $\mathrm{NaCl}(1.5 \mathrm{M})$ were dissolved in $125 \mathrm{~g}$ of $2.0 \mathrm{M} \mathrm{HCl}$ solution with stirring at room temperature. After adding TEOS, the resultant solution was hydrolyzed at $40^{\circ} \mathrm{C}$ for 0 or $2 \mathrm{~h}$ before ATMS was slowly added in. The molar composition of the mixture was $(1-x)$ TEOS: $x$ ATMS:6.1HCl:4.6NaCl:0.017P123:165H $\mathrm{H}_{2} \mathrm{O}$, where $x$ varied from 0 to 0.20 , or ATMS/(TEOS + ATMS) molar ratio being $0-20 \%$. The resulting mixture was stirred at $40{ }^{\circ} \mathrm{C}$ for $20 \mathrm{~h}$ and then transferred into a polypropylene bottle and aged at $90{ }^{\circ} \mathrm{C}$ under static condition for $24 \mathrm{~h}$. The solid product was recovered by filtration and dried at room temperature overnight. The resultant samples are denoted as SBA- $x$ ED-P-S, where $x$ is the ATMS/(TEOS + ATMS) molar ratio, $\mathrm{P}$ represents pre-hydrolysis of TEOS, and $\mathrm{S}$ represents the addition of $\mathrm{NaCl}$ salt. The template was removed from the as-synthesized material by refluxing in $95 \%$ ethanol for $24 \mathrm{~h}$. Finally, the material was filtered, washed several times with water and ethanol and dried at $50{ }^{\circ} \mathrm{C}$. A functionalized sample containing $10 \%$ ATMS with TEOS prehydrolysis in the absence of $\mathrm{NaCl}$ was denoted as SBA-10ED-P.

For comparison, $10 \mathrm{~mol} \%$ aminopropyl-functionalized SBA-15 (denoted as SBA-10NH $\mathrm{N}_{2}$ ) was prepared according to literature [23]. Four grams of Pluronic 123 was dissolved in $125 \mathrm{~g}$ of $2.0 \mathrm{M} \mathrm{HCl}$ solution at room temperature. After prehydrolysis of TEOS (7.84 g) for $2 \mathrm{~h}$, aminopropyltriethoxysilane (APTES) was slowly added into the solution. The resulting mixture was stirred at $40^{\circ} \mathrm{C}$ for $20 \mathrm{~h}$ and then transferred into a polypropylene bottle and reacted at $90{ }^{\circ} \mathrm{C}$ under static condition for $24 \mathrm{~h}$. APTES/(TEOS + APTES $)=0.1$. The template was removed by refluxing in ethanol.

\subsection{Sample characterization}

X-ray powder diffraction (XRD) patterns were obtained on a PANalytical X'Pert Pro diffractometer using $\mathrm{Cu} \mathrm{K} \alpha$ radiation $(\lambda=1.5418 \AA)$ at $45 \mathrm{kV}$ and $40 \mathrm{~mA}$. The data were collected from $0.5^{\circ}$ to $5^{\circ}(2 \theta)$ with a resolution of $0.02^{\circ}$.

$\mathrm{N}_{2}$ adsorption-desorption isotherms were measured using Micromeritics Tristar 3000 at liquid nitrogen temperature. Before the measurements, the samples were degassed at $100^{\circ} \mathrm{C}$ overnight. The specific surface areas were evaluated using Brunauer-Emmett-Teller (BET) method. Pore size distribution (PSD) was calculated using the 
Barrett-Joyner-Halenda (BJH) method based on the adsorption branch of the isotherms, and the pore size was reported from the peak position of the distribution curve. The pore volume was taken at the $P / P_{0}=0.990$ point.

Thermogravimetric (TG) analyses were carried out on a Du Pont 951 thermogravimetric analyzer with a heating speed of $10^{\circ} \mathrm{C} / \mathrm{min}$ under air in a flow of $50 \mathrm{ml} / \mathrm{min}$. N elemental analyses (EA) were performed on a Heraeus CHNS elemental analyzer. Fourier transform infrared (FTIR) was carried on a Nicolet Magna-IR 550 Spectrometer with a resolution of $2 \mathrm{~cm}^{-1}$ using the $\mathrm{KBr}$ method.

Transmission electron microscopy (TEM) was performed on a Hitachi H-7100 electron microscope, operating at $75 \mathrm{kV}$. The scanning electron microscopy was carried out on a Hitachi S-800 Electron Microscope.

The ${ }^{29} \mathrm{Si},{ }^{13} \mathrm{C}$ and ${ }^{1} \mathrm{H}$ NMR experiments were carried out at frequencies of 59.6, 75.5 and $300.1 \mathrm{MHz}$, respectively, on a Bruker DSX300 NMR spectrometer equipped with a commercial $7 \mathrm{~mm}$ MAS NMR probe. All spectra were measured at room temperature. The magic-angle spinning frequencies were set at $6 \mathrm{kHz}$ for all experiments and the variation was limited to $\pm 3 \mathrm{~Hz}$ using a commercial pneumatic control unit. Chemical shifts were externally referenced to TMS for ${ }^{29} \mathrm{Si}$ and ${ }^{13} \mathrm{C}$.

\subsection{Catalytic reactions}

Before the reaction, the functionalized SBA-15 materials were treated with methanol solution of tetramethylamonium hydroxide (TMAOH) to remove the residue $\mathrm{Cl}^{-}$ions and to neutralize the protonated amine groups. $1 \mathrm{~g}$ of the ethanol-extracted sample was suspended in $50 \mathrm{ml}$ of 0.2 $\mathrm{M}$ methanol solution of $\mathrm{TMAOH}$ at room temperature for $20 \mathrm{~min}$. The solid was recovered by filtration, washed with methanol, and finally dried at $120^{\circ} \mathrm{C}$ for one day. All the catalytic reactions were carried out in a flask with a magnetic stirrer immersed in a thermostat bath. In a typical experiment, $10 \mathrm{mmol}(1.15 \mathrm{~g})$ of ethyl cynoacetate and $10 \mathrm{mmol}(1.08 \mathrm{~g})$ of benzaldehyde were mixed in cyclohexane and kept at $35^{\circ} \mathrm{C}$, and then $0.15 \mathrm{~g}$ of the dried catalyst was rapidly added into the reactor. After the reaction, the catalyst was separated by filtration. The products were analyzed using a Chrompak CP 9000 gas chromatograph (GC) equipped with $30 \mathrm{~m} \times 0.32 \mathrm{~mm}$ RTX-50 capillary column and FID detector. $0.2 \mathrm{~g}$ of decane was used as internal standard. Individual reaction product was identified by GC-Mass spectrometry (HP6890 mass spectrometer connected with a $30 \times 0.25 \mathrm{~mm}$ RTX-50 capillary column).

\section{Results and discussion}

\subsection{Synthesis and characterization of mesoporous materials}

The functionalized materials were first prepared through one-pot synthesis without TEOS prehydrolysis. No precipitate was observed in the mixture of TEOS and ATMS at
$40{ }^{\circ} \mathrm{C}$ under acid condition. A transparent gel was formed in subsequent aging at $90{ }^{\circ} \mathrm{C}$ in case of $5 \%$ ATMS mixture. When the ATMS molar content was increased to $10 \%$, no gel or precipitation was observed even in the aging period. On the contrary, the sample synthesized with TEOS prehydrolysis such as SBA-10ED-P shows an apparent diffraction peak in the XRD pattern with a $d$-spacing of $88 \AA$, indexed to the (100) plane of hexagonal phase. These results indicate that TEOS prehydrolysis prior to the addition of ATMS is a key step for the successful synthesis of ordered diamine-functionalized SBA-15. Similar results were also observed in the synthesis of other organic-functionalized SBA-15 [11,24]. This feature was explained by that organic functional groups could disturb the assembly of the mesophase [35]. In the present case, the protonated diamine groups probably interact with the ethoxy groups of TEOS strongly through hydrogen bond so that the hydrolysis and condensation of ethoxysilane are inhibited [24]. Through prehydrolysis of TEOS, the surfactant micelles could assemble with TEOS without the perturbation from the organic functional groups.

It was reported that the addition of inorganic salt has great influence on the hydrolysis, condensation and aggregation kinetics of the silica precursors [36]. After addition of $1.5 \mathrm{M} \mathrm{NaCl}$, the SBA-10ED-P-S sample with $2 \mathrm{~h}$ prehydrolysis of TEOS shows a more intensive (100) reflection and two more well-resolved peaks indexed to (110) and (200) reflections, respectively, in comparison with SBA10ED-P synthesized without salt (Fig. 1). The $d_{100}$-spacing also increases from $88 \AA$ to $91 \AA$ (Table 1). On the other hand, for the sample synthesized with $1.5 \mathrm{M} \mathrm{NaCl}$ but without TEOS prehydrolysis, only X-ray amorphorous precipitate was obtained with very low surface area as seen in Fig. 1 and Table 1. These results further confirm that TEOS prehydrolysis prior to the addition of ATMS is essential in synthesis of ordered diamine-functionalized SBA-15.

The ATMS concentration in the reaction mixture has influence on the mesopore ordering of the materials. The XRD patterns in Fig. 1 of the materials with ATMS/ (TEOS + ATMS) molar ratio less than 0.15 and synthesized with $\mathrm{NaCl}$ show one intense (100) reflection and two well-resolved weak reflections of (110) and (200) planes, respectively. As the ATMS molar ratio increases to 0.20 , only one weak (100) reflection was observed, showing that high concentration of organic groups would reduce the mesostructure ordering. The $d_{100}$-spacing of the materials increases slightly with the increase in the ATMS content, except SBA-20ED-P-S which has low mesophase ordering. This variation in $d_{100}$-spacings is different from previous observation on the functionalized MCM-41 with triethoxyvinylsilane [37] and functionalized SBA-15 with 3-aminopropyltriethoxysilane [23]. It is generally accepted that the cell dimension of organic-functionalized mesoporous materials decreases with organic content because the strong hydrophobic interaction between the non-polar organic groups and the hydrophobic portion 

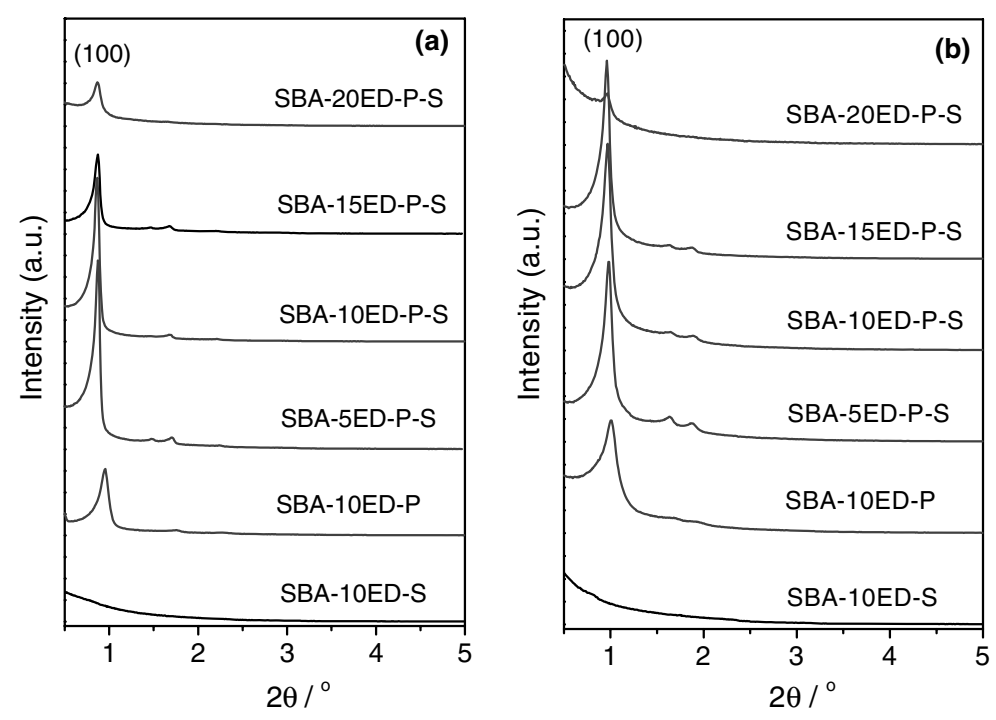

Fig. 1. XRD patterns of (a) as-synthesized and (b) extracted SBA-15 materials with different ATMS contents in the reaction mixture.

Table 1

Physico-chemical properties of the extracted functionalised SBA-15 materials containing different concentrations of ATMS

\begin{tabular}{|c|c|c|c|c|c|c|}
\hline Sample & $d_{100}$ spacing/Å & Pore diameter/Å & $S_{\mathrm{BET}} / \mathrm{m}^{2} \mathrm{~g}^{-1}$ & Pore volume $/ \mathrm{cm}^{3} \mathrm{~g}^{-1}$ & Wall thickness $\mathrm{a} / \AA$ & $N$ content $^{\mathrm{b}}$ \\
\hline SBA-10ED-S & - & - & 1.6 & 0.003 & - & $2.61(2.89)$ \\
\hline SBA-10ED-P & 88 & 58 & 316 & 0.36 & 44 & $2.17(2.89)$ \\
\hline SBA-5ED-P-S & 90 & 67 & 476 & 0.60 & 37 & $1.05(1.55)$ \\
\hline SBA-15ED-P-S & 92 & 60 & 265 & 0.33 & 46 & $2.86(4.06)$ \\
\hline SBA-20ED-P-S & 91 & 54 & 37 & 0.04 & 51 & $3.49(5.09)$ \\
\hline SBA- $10 \mathrm{NH}_{2}$ & 92 & 67 & 664 & 0.85 & 40 & $1.21(1.54)$ \\
\hline
\end{tabular}

${ }^{\text {a }}$ Calculated by $a_{\mathrm{o}}$ pore size $\left(a_{\mathrm{o}}=2 d_{100} / \sqrt{ } 3\right)$.

b $N$ content was determined by EA (theor. value) $/ \mathrm{mmol} \mathrm{g}^{-1}$.

of the surfactant molecules would draw the organic precursors into the micelles and lead to a decrease in the $d_{100^{-}}$ spacing [17]. In the diamine-functionalized SBA-15, the hydrophobic interactions between the organic moiety of ATMS and the hydrophobic PO portion of the surfactant P123 are probably interrupted by the hydrophilic diamine groups. The larger $d_{100}$-spacing observed with higher ATMS content is probably due to more hydrated water molecules are present in the interface of surfactant and silicate framework. In comparison with the as-synthesized sample, the (100) reflection peaks of the extracted materials shift slightly toward larger $2 \theta$ angles, indicating a slight shrinkage of the cell dimension upon extraction.

TEM images of the extracted functionalized materials are illustrated in Fig. 2. For diamine-functionalized SBA15 synthesized without $\mathrm{NaCl}$ in the reaction mixture, only those with less than $10 \mathrm{~mol} \%$ ATMS could have clear onedimensional channels been seen (Fig. 2(a)). In contrast, all the functionalized SBA-15 materials containing up to $20 \mathrm{~mol} \%$ ATMS displays well-ordered one-dimensional pore structure when $\mathrm{NaCl}$ was added in the synthesis mixtures. These results further confirm that inorganic salt can improve the mesostructure ordering. It is also well known that inorganic salt in the reaction mixture could influence the morphology of the mesoporous materials. Fig. 3 compares the SEM micrographs of SBA-10ED-P and SBA-10-P-S. It can be seen that SBA-10ED-P synthesized without $\mathrm{NaCl}$ consists of short rod-shaped particles of $\approx 600 \mathrm{~nm}$ in length and $300 \mathrm{~nm}$ in diameter, while SBA10ED-P-S appears to be fiber-like material with the length as long as several tens of micrometers. Moreover, these fibers of $\approx 900 \mathrm{~nm}$ in diameter are aggregated to form bundles of $\approx 6 \mu \mathrm{m}$ in diameter.

Thermal stability of the organic functionalized material was performed by TG analysis in air on the as-synthesized and ethanol-extracted SBA-10ED-P-S, and the results are shown in Fig. 4. Both samples show first weight loss of $4-7 \%$ at temperatures lower than $100^{\circ} \mathrm{C}$, corresponding to the desorption of physically adsorbed water or ethanol. This is followed by a large weight loss of $\approx 34 \%$ in the temperature range of $150-500{ }^{\circ} \mathrm{C}$ for the as-synthesized sample (Fig. 4(a)). The corresponding DTG profile displays two peaks centered at $254{ }^{\circ} \mathrm{C}$ and $347{ }^{\circ} \mathrm{C}$. The former peak is due to the thermal removal of P123 surfactant and the latter is mainly attributed to the decomposition of organic functional groups. For the sample after ethanol extraction, only one weight loss of ca. $10 \%$ was observed centered at $274{ }^{\circ} \mathrm{C}$, which is attributed to the decomposition of organic 


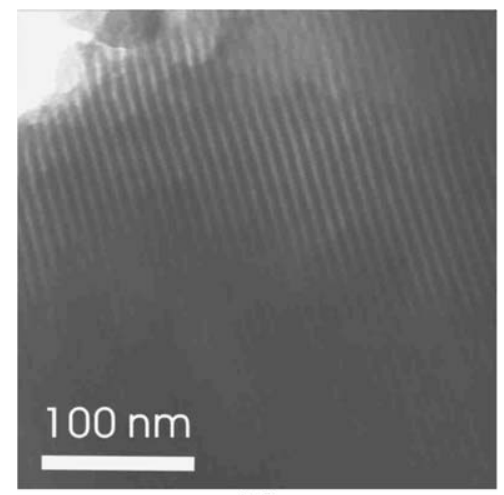

(a)

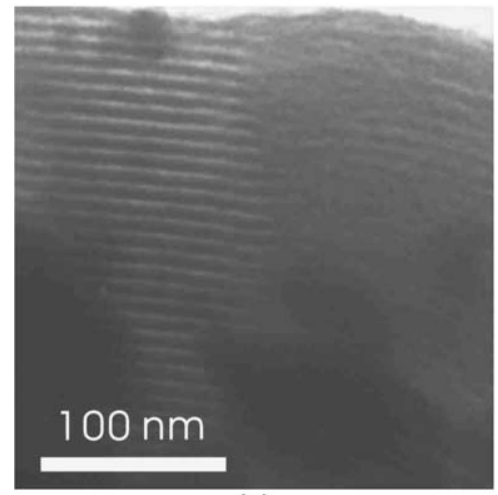

(c)

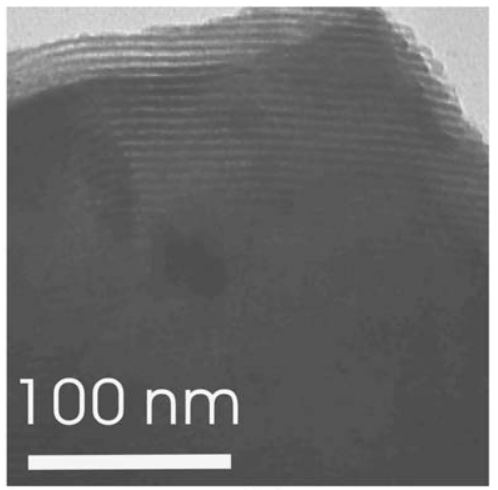

(b)

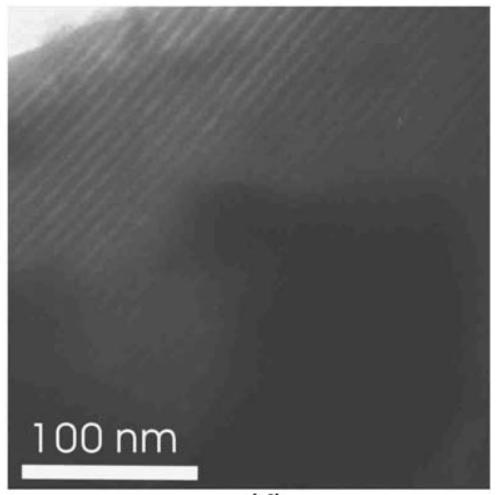

(d)

Fig. 2. TEM images of the solvent-extracted diamine-functionalized SBA-15: (a) SBA-10ED-P, (b) SBA-10ED-P-S, (c) SBA-15ED-P-S, and (d) SBA20ED-P-S.

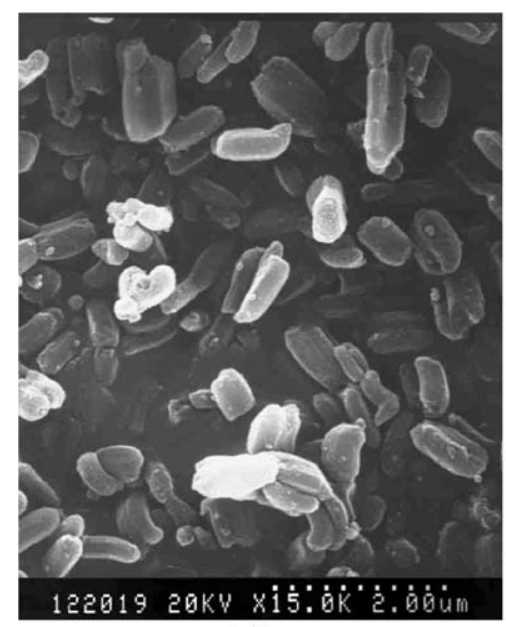

(a)

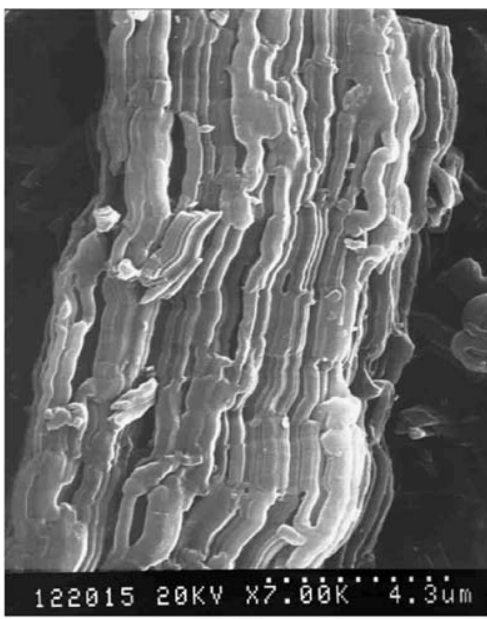

(b)

Fig. 3. SEM images of the ethanol-extracted (a) SBA-10ED-P and (b) SBA-10ED-P-S.

groups incorporated in materials. These results indicate that the P123 surfactant has been effectively removed from the as-synthesized sample through ethanol extraction. When P123 was used as the template for pure siliceous SBA-15, the block copolymer species was decomposed at around $145{ }^{\circ} \mathrm{C}$ [38]. However, in this system, the decomposition temperature of P123 surfactant is apparently elevated. On the other hand, the decomposition temperature of the organic functional groups decreased greatly from $347{ }^{\circ} \mathrm{C}$ to $274{ }^{\circ} \mathrm{C}$ after the template was extracted. These results imply that the amine groups might have a strong interaction with the surfactant. The $1-3 \%$ weight losses above $500{ }^{\circ} \mathrm{C}$ are likely due to the dehydroxylation of the silicate networks [39].

The incorporation of organic functional groups in the silica framework was also confirmed by FTIR spectra as 

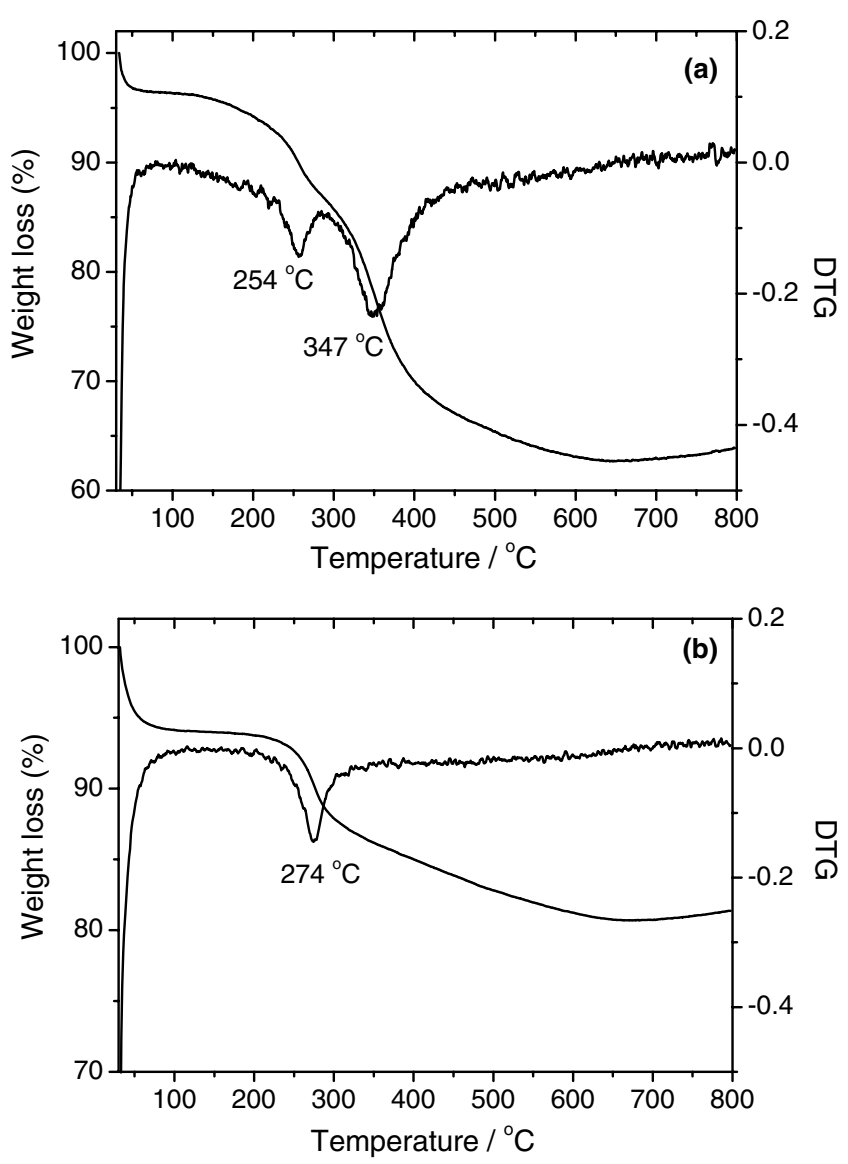

Fig. 4. TGA and DTG profiles of (a) the as-synthesized and (b) the extracted SBA-10ED-P-S.

shown in Fig. 5. The typical Si-O-Si bands around 1220, 1070,795 , and $470 \mathrm{~cm}^{-1}$ associated with the condensed silica network are observed on the as-synthesized and all the extracted samples. For the functionalized samples, a weak peak around $690 \mathrm{~cm}^{-1}$, attributed to $\mathrm{N}-\mathrm{H}$ bending vibration, and another weak peak at $1510 \mathrm{~cm}^{-1}$ corresponding to the symmetric $-\mathrm{NH}_{3}^{+}$bending vibration can be seen, showing the incorporation of amino groups. In addition, the strong peak around $1635 \mathrm{~cm}^{-1}$ is mainly assigned to $\mathrm{N}-\mathrm{H}$ bending vibration, which is overlapped with the bending vibration of adsorbed $\mathrm{H}_{2} \mathrm{O}$. The absorbance peak of the $\mathrm{C}-\mathrm{N}$ stretching vibration in the wavenumber range of $1000-1200 \mathrm{~cm}^{-1}$ cannot generally be resolved due to its overlap with the absorbance of $\mathrm{Si}-\mathrm{O}-\mathrm{Si}$ stretch in the range $1000-1130 \mathrm{~cm}^{-1}$ and that of $\mathrm{Si}-\mathrm{CH}_{2}-\mathrm{R}$ stretch in the range $1200-1250 \mathrm{~cm}^{-1}$ [21]. The weak bands around $1450-1480 \mathrm{~cm}^{-1}$, associated with $-\mathrm{CH}_{2}$ vibrations can be seen for the functionalized materials and the intensity increases with the ATMS content in the reaction mixture. This further confirms the incorporation of organic species in the framework. Similarly, the absorbance peaks associated with non-condensed $\mathrm{Si}-\mathrm{OH}$ groups in the range $940-970 \mathrm{~cm}^{-1}$ can also be observed and the peak position gradually shift toward lower wavenumber from $961 \mathrm{~cm}^{-1}$ to $942 \mathrm{~cm}^{-1}$ with the increase of ATMS concentration in

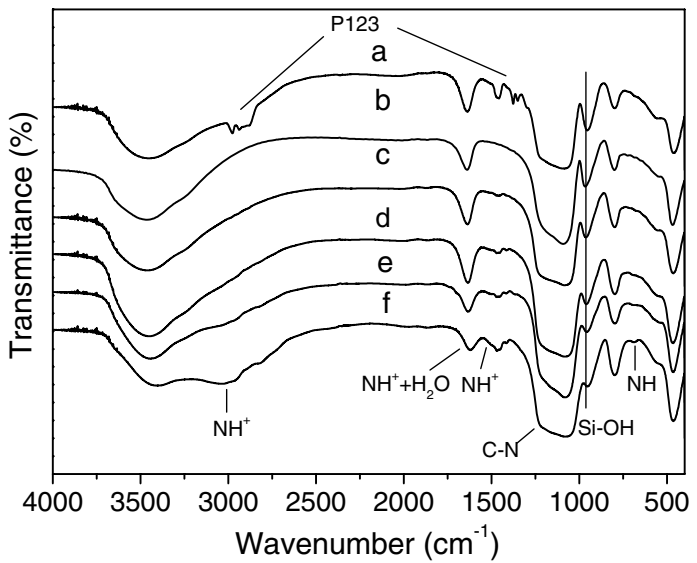

Fig. 5. FTIR spectra of (a) as-synthesized SBA-10ED-P-S and the ethanol extracted SBA-ED-P-S with different concentrations of ATMS synthesized in the presence of $\mathrm{NaCl}$ : (b) $0 \%$, (c) $5 \%$, (d) $10 \%$, (e) $15 \%$, and (f) $20 \%$.

the reaction mixture, similar to that observed on aminopropyl-functionalized SBA-15 [24]. This can be explained by the increase in interaction between the amine groups and the silanol groups through hydrogen bonding [16]. The presence of organic groups was further corroborated by a broad band in the range $2700-3400 \mathrm{~cm}^{-1}$ and by the increase of its intensity with diamine content. The absorbances in the range $3000-2700 \mathrm{~cm}^{-1}$ are due to the stretching of $-\mathrm{CH}_{2}$ groups and those in the range $3000-3400 \mathrm{~cm}^{-1}$ are assigned to the asymmetric and symmetric stretching vibrations of $\mathrm{NH}_{2}$ groups. The broadening of the silanol bands around $3480 \mathrm{~cm}^{-1}$, may be due to the cross-linking through the hydrogen-bonding interaction between the amine groups and the silanol groups. In comparison with the as-synthesized sample, the absorbance peaks associated with P123 surfactant disappear in the spectra of the extracted samples, further confirming the removal of the surfactant by ethanol extraction.

Solid state ${ }^{13} \mathrm{C}$ and ${ }^{29} \mathrm{Si}$ NMR spectroscopies were proved to be the most useful tool for providing chemical information with respect to the condensation of organosiloxane. ${ }^{29} \mathrm{Si}$ MAS NMR spectrum of the extracted SBA10ED-P-S in Fig. 6(a) displays three distinct resonance peaks at upper field corresponding to $\mathrm{Q}^{4}(\delta=-110 \mathrm{ppm})$, $\mathrm{Q}^{3}(\delta=-101 \mathrm{ppm})$ and $\mathrm{Q}^{2}(\delta=-91 \mathrm{ppm})$, and two weak peaks at lower field, assigned to $\mathrm{T}^{3}(\delta=-66 \mathrm{ppm})$ and $\mathrm{T}^{2}$ $(\delta=-58 \mathrm{ppm})$, respectively, where $\mathrm{Q}^{n}=\mathrm{Si}(\mathrm{OSi})_{n}(\mathrm{OH})_{4-n}$, $n=2-4$ and $\mathrm{T}^{m}=\mathrm{RSi}(\mathrm{OSi})_{m}(\mathrm{OH})_{3-m}, \quad m=1-3$. The appearance of $\mathrm{T}^{m}$ peaks confirms that the organosiloxane precursor is condensed as part of the silicate framework. The relative integrated intensity of the $\mathrm{T}^{m}$ and $\mathrm{Q}^{n}$ signals $\left(\mathrm{T}^{m} /\left(\mathrm{T}^{m}+\mathrm{Q}^{n}\right)\right)$ is 0.090 , which is close to the expected value of 0.10 on the basis of the composition of AEAPTMS in the reaction mixture. The ${ }^{13} \mathrm{C}$ CP-MAS NMR spectrum of SBA-1-ED-P-S in Fig. 6(b) clearly shows five resonance peaks at $10,21,38,47$ and $52 \mathrm{ppm}$, corresponding to the $\mathrm{C}$ atoms on the $\mathrm{Si}-\mathrm{CH}_{2}-\mathrm{CH}_{2}-\mathrm{CH}_{2}-\mathrm{NH}-\mathrm{CH}_{2}-\mathrm{CH}_{2}-$ $\mathrm{NH}_{2}$ group in sequence from left to right, respectively [18]. This result further confirms that ATMS precursors 

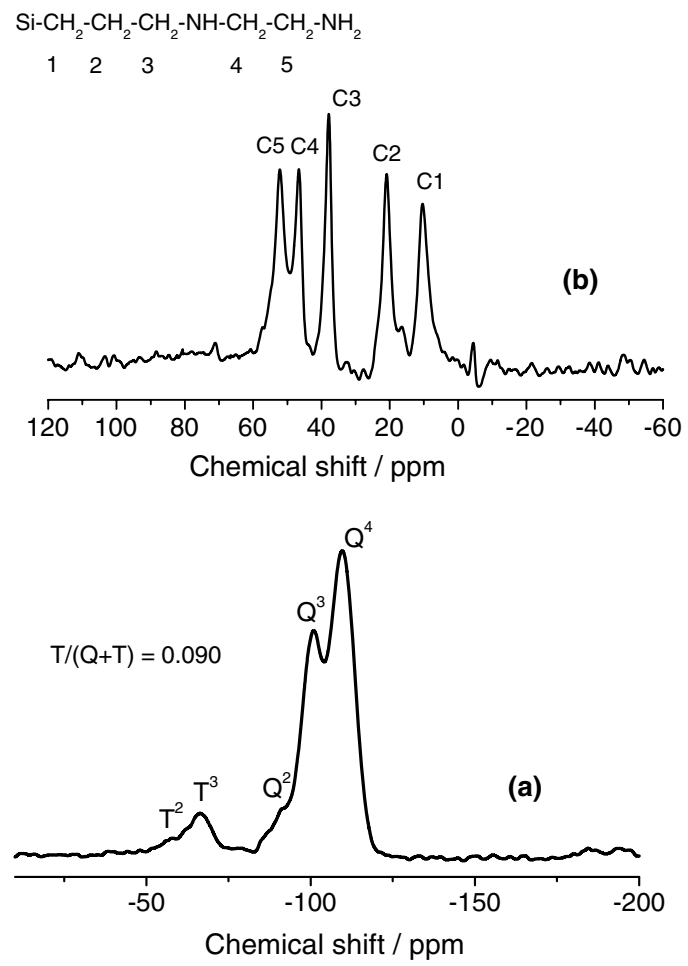

Fig. 6. (a) ${ }^{29} \mathrm{Si}$ MAS NMR spectrum and (b) ${ }^{13} \mathrm{C}\{1 \mathrm{H}\}$ CPMAS NMR spectrum of the extracted SBA-15 with $10 \%$ ATMS synthesized in the presence of $\mathrm{NaCl}$.

are co-condensed into the mesoporous silca and the organic moieties are not decomposed during the preparation procedure. Besides, no resonance peaks corresponding to the surfactant P123 were observed in the range of $67-77 \mathrm{ppm}$, indicating that the surfactant was completely removed during the extraction as also supported by TG and IR spectra.

Chemical elemental analysis of nitrogen was used to quantitatively determine the incorporation of amine functional groups. Based on the results in Table 1, about 70\% of the ATMS precursor was effectively incorporated in the silica framework. The incorporation percentages are lower than $\approx 80 \%$ for aminopropyl-functionalized SBA- 15 [24], implying that the diamine groups are more seriously interfering the assembly of mesostructure than the single amine groups. As a result, the N content of SBA-10ED$\mathrm{P}-\mathrm{S}$ is lower than twice the amount of SBA- $10 \mathrm{NH}_{2}$. Nevertheless, the $\mathrm{N}$ content could reach as high as $3.49 \mathrm{mmol} \mathrm{g}^{-1}$ when the ATMS/(TEOS + ATMS) molar ratio in the initial mixture was 0.2 , although the mesostructure ordering was not good and the surface area was low for the material with such a high $\mathrm{N}$ content. In comparison of the materials synthesized with and without TEOS prehydrolysis (SBA$10 \mathrm{ED}-\mathrm{S}$ versus SBA-10ED-P-S), it is noticed that the amine content decreases with TEOS prehydrolysis. In contrast, the addition of inorganic salt does not affect the amine content significantly.

$\mathrm{N}_{2}$ adsorption-desorption isotherms of the extracted functionalized materials are illustrated in Fig. 7(a). The SBA-10ED-S sample synthesized without TEOS prehy-
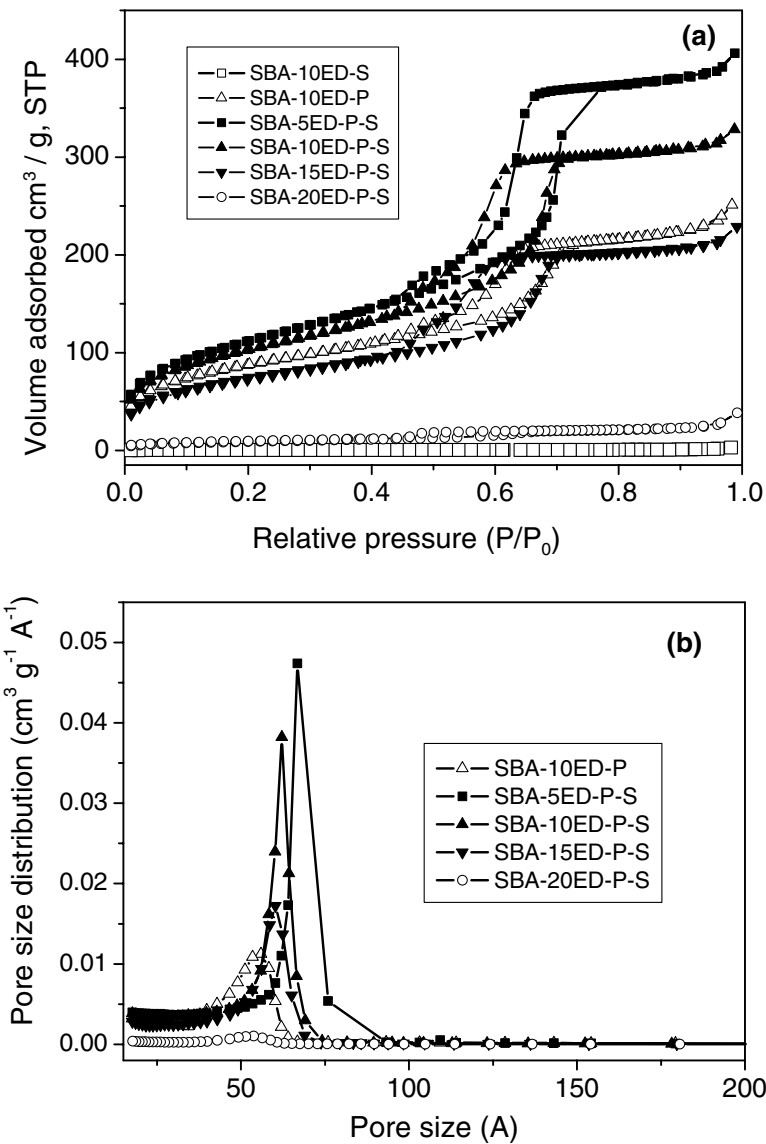

Fig. 7. (a) Nitrogen adsorption-desorption isotherms and (b) BJH pore size distribution plots of the extracted functionalized materials with different ATMS contents in the reaction mixture.

drolsis shows negligible amount of nitrogen adsorption. All the other samples with TEOS prehydrolysis containing ATMS/(TEOS + ATMS) molar ratio less than 0.15 displays type IV isotherms with steep increases in adsorption at relative pressure $P / P_{0}$ around $0.6-0.8$, similar to that of pure SBA-15. As expected for organic-functionalized mesoporous silica, the BET surface area, BJH pore sizes and pore volumes of the materials decrease gradually with ATMS content, due to the occupation of large organic groups in the pore channels. On the contrary, the wall thickness of the materials increases with ATMS content. BJH pore size analysis shows that the materials synthesized with TEOS prehydrolysis and the aid of $\mathrm{NaCl}$ contain mesopores of narrow pore size distribution (PSD) with diameters in $60-67 \AA$ range. In contrast, sample SBA-10ED-P which was synthesized without $\mathrm{NaCl}$ shows a broader PSD and smaller pore diameter (Fig. 7(b)). Therefore, the inorganic salt $\mathrm{NaCl}$ not only influences the mesostructure ordering and the morphology of the materials, but also enhances the BET surface area, pore size and pore volume. When the ATMS concentration further increases to a ratio of 0.20 , a very small hysteresis loop was observed, and that was attributed to poor assembly of the mesostructure in high ATMS concentration. 


\subsection{Catalysis reactions}

The Knoevenagel condensation is of great importance to the synthetic chemists in the construction of new $\mathrm{C}-\mathrm{C}$ bonds [40]. Amine-functionalized silica has been found to be effective base catalysts for this synthesis [14]. Here we choose the Knoevenagel reaction of benzaldehyde with ethyl cynoacetate (Scheme 1) to form $\alpha, \beta$-unsaturated compound (2-cyano-3-phenyl-acrylic acid ethyl ester) as a probe reaction to examine the catalytic performance of the obtained materials. Before the reaction, the diaminefunctionalized materials were treated with $0.2 \mathrm{M}$ methanol solution of TMAOH to remove the residue $\mathrm{Cl}^{-}$ions and to neutralize the protonated amine groups in the samples. The analysis showed that the physical properties, texture and amine contents of the catalysts after treatment with TMAOH were almost unchanged. Pure siliceous SBA-15 treated under the same condition showed no catalytic activity. Fig. 8 gives the dependency of the yields of $\alpha, \beta$-unsaturated compound on the reaction period over various amine-functionalized SBA-15 materials. No other side products except the $\alpha, \beta$-unsaturated compound were detected in the products based on the analysis by $\mathrm{GC}-$ MS chromatography. All the catalysts showed rapid reaction rates in the first $150 \mathrm{~min}$, after this the yields of the product only increased slightly. This might be due to the deactivation of the catalysts by adsorption of formed water and a trace amount of amide product [14]. For the catalysts
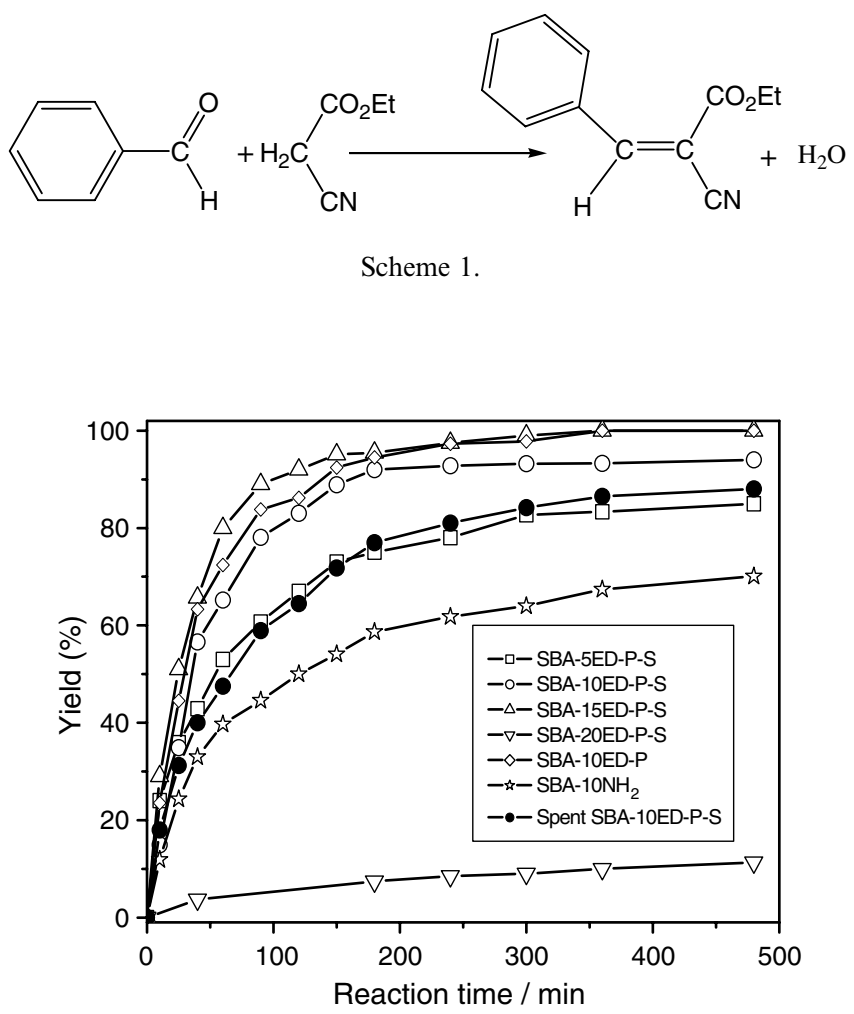

Fig. 8. Knoevenagel reaction of benzaldehyde with ethyl cyanoacetate in cyclohexane over different diamine-functionalized SBA-15 catalysts. with ATMS/(TEOS + ATMS) molar ratio less than 0.15, the product yields increase with the nitrogen content in the catalysts. As the ATMS concentration was further increased to 0.20 , the product yield decreased markedly due to the low surface area and pore volume of the material. It can be seen in Fig. 8 that the product yield over SBA-10ED-P-S is slightly lower than that over SBA10ED-P. Both contained similar loadings of amino groups, but the latter was prepared without salt and had poor $\mathrm{X}$-ray mesostructure ordering. This might be because the reactants could access the catalytic sites more easily by diffusion as the pore channels of SBA-10ED-P catalyst were probably shorter than those of SBA-10ED-P-S.

Since each diamine functional group contains two amine sites but aminopropyl group contains only one amine site, it is expected that aminopropyl-functionalized SBA-10 $\mathrm{NH}_{2}$ catalyst should have similar amine sites as diamine-functionalized SBA-5ED-P-S catalyst. However, analysis shows that single amine-functionalized $\mathrm{SBA}-10 \mathrm{NH}_{2}$ has higher surface area, larger pore volume and higher $\mathrm{N}$ content than SBA-5ED-P-S. Nevertheless, the catalytic activity of SBA$10 \mathrm{NH}_{2}$ is lower than the diamine-functionalized SBA-5EDP-S as shown in Fig. 8. The discrepancy is so marked that it is proposed that two amine sites on the diamine group might have synergistic effect to some extent. The detailed mechanism will need further investigation.

The spent SBA-10ED-P-S was treated with methanol in a Soxhlet apparatus for $3 \mathrm{~h}$ and then dried at $120^{\circ} \mathrm{C}$ overnight before tested as a catalyst again. The spent catalyst gave only slightly lower activity than the fresh catalyst ( $85 \%$ versus $93 \%$ yield), indicating that most of the active sites could be regenerated by a simple solvent treatment.

\section{Conclusions}

Highly-ordered large pore diamine-functionalized SBA15 materials with high loadings of amino groups (up to $3.49 \mathrm{mmol} \mathrm{g}^{-1}$ ) were synthesized under strong acid condition by the combination of TEOS prehydrolysis and addition of inorganic salt $\mathrm{NaCl}$. Prehydrolysis of TEOS prior to the addition of ATMS precursors was a key step for the successful synthesis of ordered functionalized materials. Inorganic salt could enhance the mesostructure ordering and influence the morphology of the materials. The diamine-functionalized SBA-15 showed better catalytic performance than aminopropyl-functionalized counterpart in the Knoevenagel reaction of benzaldehyde with ethyl cynoacetate to form $\alpha, \beta$-unsaturated compounds in liquid phase.

\section{Acknowledgment}

The authors acknowledge the financial supports from the National Science Council, Taiwan. Acknowledgements are also extended to Mr. C.-Y. Tang, Ms. C.-Y. Lin and Ms. G.-W. Lu of National Taiwan University for TEM, SEM experiments and elemental analysis. 


\section{References}

[1] C.T. Kresge, M.E. Leonowicz, W.J. Roth, J.C. Vartuli, J.S. Beck, Nature 359 (1992) 710.

[2] I. Rodriguez, S. Iborra, A. Corma, F. Rey, J.L. Jordá, Chem. Commun. (1999) 593.

[3] A. Stein, Adv. Mater. (Weinheim. Ger.) 15 (2003) 763.

[4] I.K. Mbaraka, D.R. Radu, V.S.-Y. Lin, B.H. Shanks, J. Catal. 219 (2003) 329.

[5] X.G. Wang, C.C. Chen, S.-Y. Chen, Y. Mou, S. Cheng, Appl. Catal. A: Gen. 281 (2005) 47.

[6] S. Dai, M.C. Burleigh, Y. Shin, C.C. Morrow, C.E. Barnes, Z. Xue, Angew. Chem., Int. Ed. 38 (1999) 1235.

[7] H. Hata, S. Saeki, T. Kimura, Y. Sugahara, K. Kuroda, Chem. Mater. 11 (1999) 1110.

[8] V.S.Y. Lin, C.Y. Lai, J. Huang, S.A. Song, S. Xu, J. Am. Chem. Soc. 123 (2001) 11510.

[9] W. Zhou, J.M. Thomas, D.S. Shephard, B.F.G. Johnson, D. Ozkaya, T. Maschmeyer, R.G. Bell, Q. Ge, Science 280 (1998) 705.

[10] W.H. Zhang, X.B. Lu, J.H. Xiu, Z.L. Hua, L.X. Zhang, M. Robertson, J.L. Shi, D.S. Yan, J.D. Holmes, Adv. Funct. Mater. 14 (2004) 544.

[11] D. Margollese, J.A. Melero, S.C. Christianesn, B.F. Chmelka, G.D. Stucky, Chem. Mater. 12 (2000) 2448.

[12] K.A. Koyano, T. Tatsumi, Y. Tanaka, S. Nakata, J. Phys. Chem. B 101 (1997) 9436

[13] M.H. Lim, A. Stein, Chem. Mater. 11 (1999) 3285.

[14] D.J. Macquarrie, D.B. Jackson, Chem. Commun. (1997) 1781.

[15] M.T. Drexler, M.D. Amiridis, J. Catal. 214 (2003) 136.

[16] G. Sartori, F. Bigi, R. Maggi, R. Sartorio, D.J. Macquarrie, M. Lenarda, L. Storaro, S. Coluccia, G. Martra, J. Catal. 222 (2004) 410.

[17] T. Yokoi, H. Yoshitake, T. Tatsumi, J. Mater. Chem. 14 (2004) 951.

[18] S. Huh, J.W. Wiench, J.C. Yoo, M. Pruski, V.S.Y. Lin, Chem. Mater. 15 (2003) 4274.

[19] D.J. Macquarrie, D.B. Jackson, J.E.G. Mdoe, J.H. Clark, New J. Chem. 23 (1999) 539.
[20] A.S.M. Chong, X.S. Zhao, J. Phys. Chem. B 107 (2003) 12650.

[21] H.H.P. Yiu, P.A. Wright, N.P. Botting, J. Mol. Catal. B: Enzym. 15 (2001) 81

[22] Y.J. Han, G.D. Stucky, A. Butler, J. Am. Chem. Soc. 121 (1999) 9897.

[23] X.G. Wang, K.S.K. Lin, J.C.C. Chan, S. Cheng, Chem. Commun. (2004) 2762.

[24] X.G. Wang, K.S.K. Lin, J.C.C. Chan, S. Cheng, J. Phys. Chem. B 109 (2005) 1763.

[25] X. Wang, Y.-H. Tseng, J.C.C. Chan, S. Cheng, Micropor. Mesopor. Mater. 85 (2005) 241.

[26] J.M. Kim, S.K. Kim, R. Ryoo, J. Phys. Chem. B 103 (1999) 6200.

[27] C. Yu, B. Tian, J. Fan, G.D. Stucky, D. Zhao, J. Am. Chem. Soc. 124 (2002) 4556.

[28] B.L. Newalkar, S. Komarneni, Chem. Mater. 13 (2001) 4573.

[29] Y. Wang, B. Zibrowius, C. Yang, B. Spliethoff, F. Schüth, Chem. Commun. (2004) 46.

[30] W.H. Zhang, J.L. Shi, H.R. Chen, Z.L. Hua, D.S. Yan, Chem. Mater. 13 (2001) 648.

[31] C. Yang, P.H. Liu, Y.F. Ho, C.Y. Chiu, K.-J. Chao, Chem. Mater. 15 (2003) 275.

[32] J.L. Gu, J.L. Shi, G.J. You, L.M. Xiong, S.X. Qian, Z.L. Hua, H.R. Chen, Adv. Mater. 17 (2005) 557.

[33] H. Zhu, B. Lee, S. Dai, S.H. Overbury, Langmuir 19 (2003) 3974.

[34] D. Jiang, Q. Yang, J. Yang, L. Zhang, G. Zhu, W. Su, C. Li, Chem. Mater. 17 (2005) 6154.

[35] J.A. Melro, G.D. Stucky, R. van Grieken, G. Morales, J. Mater. Chem. 12 (2002) 1664

[36] Y.Q. Wang, C.M. Yang, B. Zibrowius, B. Spliethoff, M. Linden, F. Schuth, Chem. Mater. 15 (2003) 5029.

[37] Y. Mori, T.J. Pinnavaia, Chem. Mater. 13 (2001) 2173.

[38] D. Zhao, J. Feng, Q. Huo, N. Melosh, G.H. Frodrickson, B.F. Chmelka, G.D. Stucky, Science 279 (1998) 548.

[39] X.S. Zhao, G.Q. Lu, A.K. Whittaker, G.J. Millar, H.Y. Zhu, J. Phys. Chem. B 101 (1997) 6525.

[40] G. Jones, Org. React. 15 (1967) 204. 